\title{
A comparison between three commercial kits for chromogranin A measurements
}

\author{
M Stridsberg, B Eriksson, K Öberg and E T Janson \\ Department of Medical Sciences, Clinical Chemistry, Uppsala University Hospital, S-751 85 Uppsala, Sweden \\ (Requests for offprints should be addressed to M Stridsberg; Email: mats.stridsberg@clm.uas.lul.se)
}

\begin{abstract}
Chromogranin (CgA) has been shown to be an excellent marker for neuroendocrine tumours. There are now three commercial assays on the market. We wanted to compare the usefulness of the different kits in a clinical situation. We have thus measured CgA in 77 patients and compared the results from the different methods. CgA was measured with three different commercial kits according to the recommendations from the manufacturers (CGA-RIA CT; CIS bio international, Gif-sur-Yvette cedex, France, DAKO Chromogranin A ELISA kit; DAKO A/S, Glostrup, Denmark and CgA; EuroDiagnostica, Malmö, Sweden). The sensitivity and specificity differed between the different kits. The CIS kit showed a sensitivity of $67 \%$
\end{abstract}

and a specificity of $96 \%$. The sensitivity and specificity were both $85 \%$ for the DAKO kit and $93 \%$ and $88 \%$ respectively for the EuroDiagnostica assay. We have concluded that $\mathrm{CgA}$ is an important tumour marker for all neuroendocrine tumours. However, different analytical properties of the respective kits give different performances, a fact that must be taken into consideration when comparing results from different clinical studies. A recognised international standard for $\operatorname{CgA}$ would be a step on the way to harmonisation, but antibody selection and construction of the assays will probably still influence the results.

Journal of Endocrinology (2003) 177, 337-341

\section{Introduction}

Chromogranin A (CgA) is an acidic glycoprotein with 439 amino acids and a molecular mass of $48 \mathrm{kDa}$, occurring in the secretory granules of most neuroendocrine cell types. $\mathrm{CgA}$ is released together with peptide hormones and neuropeptides upon stimulation. $\mathrm{CgA}$ is also released from neuroendocrine-derived tumours. Thus, CgA has been shown to be an excellent marker for neuroendocrine tumours and has been used as a tumour marker since the middle of the 1980s. Several assays for measurements of intact $\mathrm{CgA}$ and the different cleavage products have been developed and used both for research and for clinical purposes (for an overview of methods see Stridsberg 2000). During recent years, an increasing appreciation of $\mathrm{CgA}$ has been noted and the number of $\mathrm{CgA}$ measurements is increasing. Also, the CgA analysis has become more accessible since there are now three commercial assays on the market.

The primary structure of human CgA contains 10 pairs of basic amino acids, which are potential cleavage sites for specific endogenous proteases, but also other sites in the molecule can be subjected to cleavage. Several CgA-related peptides have been identified in biological tissue from humans and other species (Laslop et al. 2000). Different neuroendocrine cells can process
CgA differently (Portela-Gomes \& Stridsberg 2001). Chromatographic separations of tissue extracts have shown that the extent of $\mathrm{CgA}$ processing appears to vary in different neuroendocrine tissues, with a more extensive cleavage of $\mathrm{CgA}$ in pancreatic islets than in the adrenals (Iacangelo \& Eiden 1995). Tumours can also release different molecular forms of $\mathrm{CgA}$. Thus, $\mathrm{CgA}$ released from different neuroendocrine tumours can be expected to exist in different molecular forms. Due to the construction of the CgA assay, i.e. the antibody-binding epitopes, different methods can give different results.

The three commercially available CgA kits are somewhat different in composition, have different standardisation and use different antibodies. One of the methods uses monoclonal antibodies, while the other two use polyclonal antibodies. One uses enzyme detection (enzyme-linked immunosorbent assay; ELISA) and the other two are radioimmunoassays. Also, the assays use different sources of standards and express the results in three different ways (ng/ml, U/l and nmol/l). Thus it is likely that the results from the measurements in the different kits can differ substantially. We wanted to compare the usefulness of the different kits in a clinical situation. We have thus measured $\mathrm{CgA}$ in 77 patients and compared the results from the different methods. 


\section{Materials and Methods}

\section{Patients and blood samples}

Patients were recruited consecutively from a national referral centre for neuroendocrine tumours. The first 77 patients arriving during the first 3 months of 2002 were enrolled in the study. CgA was measured with three commercial CgA assays (see below). The patients represented a variety of diagnoses, from known neuroendocrine tumours on different treatments to new cases submitted for investigation. The study was approved by the local ethical committee at the Uppsala University Hospital.

\section{Sample collection and CgA methods}

Blood was collected in chilled heparinised vacutainer tubes and centrifuged at $3000 \mathrm{~g}$ within $30 \mathrm{~min}$. Plasma was frozen in aliquots and kept at $-20{ }^{\circ} \mathrm{C}$ before analysis in the different assays.

$\mathrm{CgA}$ was measured with three different commercial kits according to the recommendations from the manufacturers (CGA-RIA CT; CIS bio international, Gifsur-Yvette cedex, France, DAKO Chromogranin A ELISA kit; DAKO A/S, Glostrup, Denmark and CgA; EuroDiagnostica, Malmö, Sweden). Hereafter the kits are referred to as CIS, DAKO and ED respectively.

\section{Statistics}

Correlation between different assays was calculated from linear regression.

\section{Results}

\section{Patients}

This study comprised 77 patients, where 46 patients had clinically manifest neuroendocrine tumours, indicated by the presence of primary tumours or metastases that could be radiologically verified. Of these, 16 had endocrine pancreatic tumours, 14 had foregut carcinoid tumours, 11 had midgut carcinoid tumours, one had a hindgut carcinoid tumour and four had other tumours with neuroendocrine differentiation. In total, 31 patients lacked signs of neuroendocrine tumours. Of these, 17 patients had been operated upon with radical removal of primary tumours and/or metastases and are thus considered tumour free. Furthermore, 11 patients were referred for clinical evaluation but were considered free from tumours. Three patients had adrenocortical cancers. Thus, based on the clinical presentation, 46 patients had neuroendocrine tumours and were expected to show elevated levels of $\mathrm{CgA}$ and 31 patients were expected to have normal levels.

With the CIS kit, blood levels of CgA ranged from 20 to $1200 \mu \mathrm{g} / 1$. In total, 42 patients had normal levels, i.e.
$<99 \mu \mathrm{g} / 1$, and 35 had elevated levels. Similarly, the range in the DAKO kit was $7 \cdot 3$ to $500 \mathrm{U} / 1$, where 31 patients had normal levels, i.e. <19 U/1, and 46 had elevated levels. Levels of CgA in the ED kit ranged from $2 \cdot 1$ to $880 \mathrm{nmol} / 1$, where 26 patients had normal levels, i.e. $<4.1 \mathrm{nmol} / 1$, and $51 \mathrm{had}$ elevated levels. There was a significant correlation between the respective kits; however, the DAKO and ED kits showed closer correlation than the CIS kit (Fig. 1 and Table 1).

Within the group of patients without neuroendocrine tumours there were some differences between the different CgA assays (Fig. 1). Of the three patients with adrenocortical cancers all showed elevated CgA levels with the ED method, but only one had elevated levels in the other two kits. In the group of 28 patients without neuroendocrine tumours, there were two patients on long-standing treatment with proton-pump inhibitors (PPI), these had elevated levels of $\mathrm{CgA}$ in all methods. Since this treatment is known to cause falsely elevated plasma CgA, these patients were excluded from the calculations of sensitivity and specificity. Of the remaining 72 patients, only one had elevated levels of CgA with the CIS assay, but four had falsely elevated levels with the DAKO assay and three with the ED assay. Of these, one patient with a plasma sample contaminated with haemolysis had elevated $\mathrm{CgA}$ in the DAKO method, but not in the CIS and ED methods. None of the patients had signs of impaired renal function, which is also a known cause of elevated plasma CgA levels. Thus, the specificity of the different assays was $96 \%, 85 \%$ and $88 \%$ for the CIS, DAKO and ED kits respectively.

Of the patients with clinically manifest neuroendocrine tumours, the overall sensitivity of the different kits was $67 \%, 85 \%$ and $93 \%$ respectively for the CIS, DAKO and ED kits. Also, within the different neuroendocrine diagnostic groups, there were some differences between the different CgA assays (Fig. 1 and Table 1). In the group of 16 patients with endocrine pancreatic tumours, 11 (69\%) showed elevated levels of CgA in the CIS method, while $13(81 \%)$ did in the DAKO and 14 (88\%) in the ED methods. Similarly, with the 14 patients with foregut carcinoid tumours, only nine (64\%) had elevated CgA levels with the CIS method, while $12(86 \%)$ had elevated levels with the DAKO assay and $14(100 \%)$ with the ED assay. Within the group of 11 patients with midgut carcinoid tumours, eight patients $(73 \%)$ had elevated levels of CgA with the CIS assay and ten (91\%) patients had elevated levels with the DAKO and ED assays. Two (50\%) of the four patients with neuroendocrine differentiated tumours had elevated levels of CgA in the CIS assay, while three $(75 \%)$ had elevated levels in the DAKO assay and all four $(100 \%)$ had increased levels of $\mathrm{CgA}$ in the ED method. The sensitivity, specificity, positive predictive value and negative predictive value of the three assays are given in Table 2. 

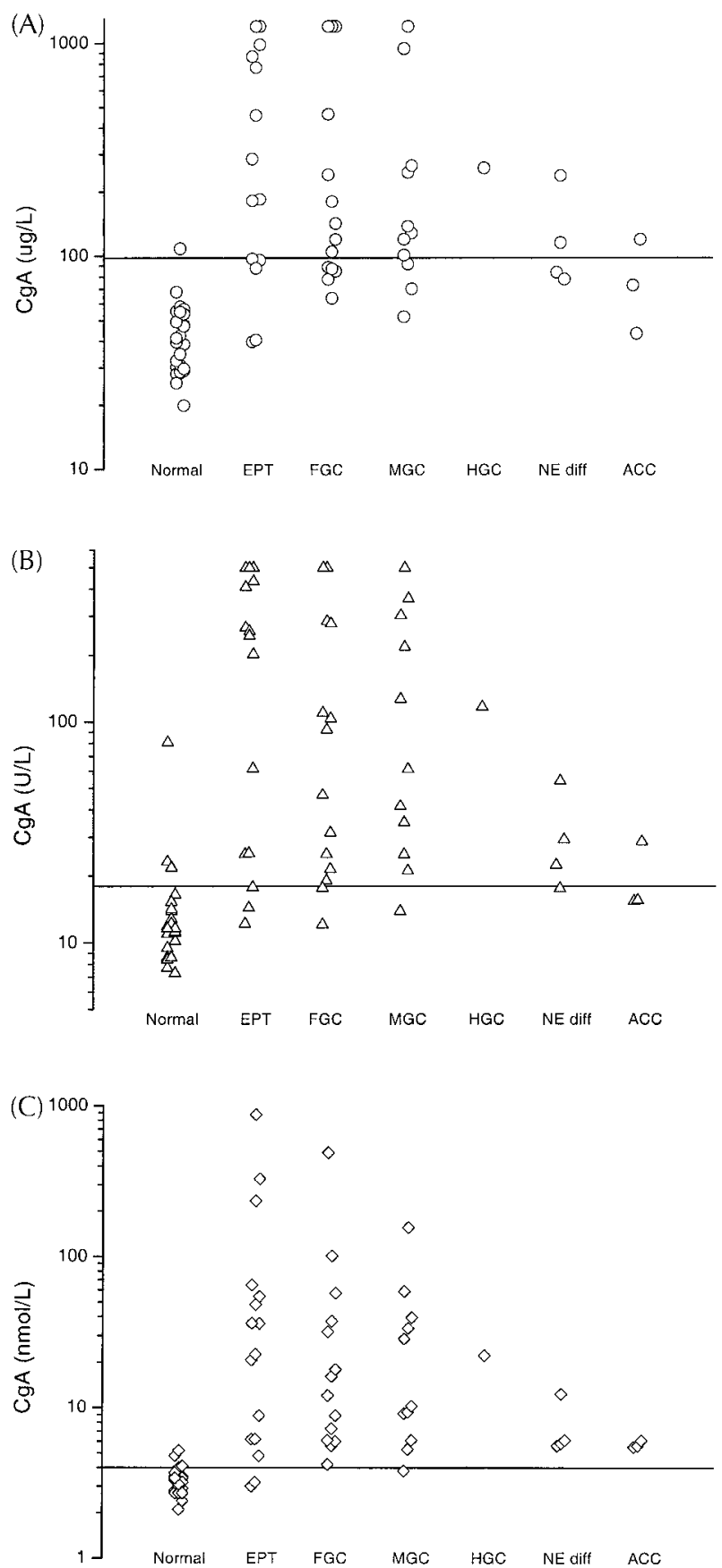

Figure 1 The measured plasma CgA concentrations in the three commercial CgA kits. The values are presented in relation to the diagnosis and the horizontal lines indicate the upper reference limit. (A) CIS, (B) DAKO and (C) ED. Normal=patients with non-neuroendocrine diseases, EPT =endocrine pancreatic tumours, $\mathrm{FGC}=$ foregut carcinoid tumours, $\mathrm{MGC}=$ midgut carcinoid tumours, $\mathrm{HGC}=$ hindgut carcinoid tumours, $\mathrm{NE}$ diff $=$ neuroendocrine differentiated tumours, $\mathrm{ACC}=$ adrenocortical cancers. The sensitivity of the different assays for each type of neuroendocrine tumour type is given in Table 1.
Table 1 The percentage of patients with increased levels of CgA in the three commercial kits. The results are given in relation to the different diagnostic groups: $\mathrm{CIS}, \mathrm{DAKO}$ and ED

No tumour, $n=26$

Adrenocortical cancers, $n=3$

Endocrine pancreatic tumours, $n=16$

Foregut carcinoids, $n=14$

Midgut carcinoids, $n=11$

Hindgut carcinoids, $n=1$

Neuroendocrine differentiated tumours, $n=4$

Total no. of patients with neuroendocrine

tumours, $n=46$

\begin{tabular}{|c|c|c|}
\hline CIS & DАKO & ED \\
\hline 4 & 15 & 1 \\
\hline 33 & 33 & 100 \\
\hline 69 & 81 & 8 \\
\hline 6 & 86 & 100 \\
\hline 7 & 91 & 91 \\
\hline 100 & 100 & 100 \\
\hline 50 & 75 & 100 \\
\hline & 85 & \\
\hline
\end{tabular}

\section{Discussion}

CgA has become the most important circulating tumour marker for neuroendocrine tumours (Nobels et al. 1997, Bajetta et al. 1999, Giovanella et al. 1999, Franke et al. 2000). CgA levels are increased in most patients with carcinoid tumours (foregut and midgut), endocrine pancreatic tumours of different syndromes, pheochromocytoma, neuroblastoma and small cell lung cancers (Nobels et al. 1998). Several in-house methods for the determination of CgA have been presented previously (Stridsberg 2000). Today, three commercial assays are available and these are compared in the present study. However, the lack of any international standardisation makes it difficult to compare results from the different $\mathrm{CgA}$ assays and thus compare clinical responses to various treatments, especially in multicentre-based clinical trials. In this study, we wanted to investigate the assays under clinical relevant conditions, i.e. not only measure $\mathrm{CgA}$ in patients with known neuroendocrine tumours, but also include patients that were under consideration for suspected tumours and patients that were not expected to have increased levels of CgA. Thus, 77 consecutively recruited patients from a national referral unit for neuroendocrine tumours were enrolled in the study.

The three commercial CgA kits differ in at least two aspects. First, the antibodies used in the CIS kit are monoclonal antibodies directed against the $\mathrm{CgA}$ amino acid sequences 145-197 and 198-245. Since one of the

Table 2 The sensitivity (SENS), specificity (SPEC), positive predictive value (PPV) and negative predictive value (NPV) for the three commercial kits: $\mathrm{CIS}, \mathrm{DAKO}$ and $\mathrm{ED}$

\begin{tabular}{|c|c|c|c|c|}
\hline & SENS & SPEC & PPV & NPV \\
\hline $\mathrm{CIS}$ & 67 & 96 & 97 & 63 \\
\hline DAKO & 85 & 85 & 91 & 76 \\
\hline ED & 93 & 88 & 93 & 88 \\
\hline
\end{tabular}

Journal of Endocrinology (2003) 177, 337-341 
antibodies is iodinated, this gives a sandwich radioimmunoassay, which can detect CgA molecules providing that both the antibody-binding sequences are present in the same molecule. The DAKO assay does not give full information on its construction, but it appears that the kit uses one or two polyclonal antibodies directed against $\mathrm{CgA}$. Since one subset of antibodies is coupled to an enzyme (peroxidase), this approach gives a sandwich ELISA method. The ED method uses one polyclonal antibody directed against the CgA 116-439 amino acid sequence. In this assay the standard is iodinated, which gives a classical competitive radioimmunoassay.

Secondly, all three assays use different standardisation. The CIS kit uses a recombinant human CgA produced in E. coli (Taupenot et al. 1995), and the concentration is expressed in $\mathrm{ng} / \mathrm{ml}$. The DAKO assay, however, uses a $23 \mathrm{kDa}$ C-terminal fragment of CgA. Apparently the true concentration of the molecule is unknown since the manufacturer expresses the results in units/l. On the other hand, the ED kit uses a CgA fraction purified from urine from patients with carcinoid tumours. In this case, the molecule is well characterised, covers the $\mathrm{CgA}$ amino acid sequence 116-439, and is expressed in nmol/1 (Stridsberg et al. 1993). Since all three assays use different sources of standards and are standardised differently, the comparison between the kits had to rely upon the respective reference interval given by the manufacturer.

It has previously, by biochemical separation methods, been shown that the rate of $\mathrm{CgA}$ processing can differ in different tissue. Thus, processing of CgA seems to be more extensive in endocrine pancreatic islet cells and in the small intestine as compared with endocrine cells in the adrenal medulla and neuronal cells (Curry et al. 1990, Borglum Jensen et al. 1991). A further processing diversity is likely to be found in different neuroendocrine tumours, which has been indicated by immunohistochemical findings (Portela-Gomes et al. 2001). This different processing in tumour-derived $\mathrm{CgA}$, in combination with the different constructions of the commercial kits, probably determines the different results demonstrated in the study.

The ED kit showed the highest sensitivity (93\%). Considering the construction of the assay, this was expected. A competitive assay using polyclonal antibodies should have the ability to detect more antibody-binding epitopes than a non-competitive assay since the latter must have a defined $\mathrm{CgA}$ fragment, which can bind to both of its antibodies. This is not the situation for a competitive assay, which is likely to detect different molecular forms of $\mathrm{CgA}$. Another factor that influences the sensitivity is the definition of the upper reference limit. For the CIS kit it seems that the reference limit is too high since the sensitivity is low (67\%) but the specificity is high (96\%) compared with the DAKO assay, which showed $85 \%$ sensitivity and specificity. However, the competitive ED assay showed both higher sensitivity (93\%) and specificity $(88 \%)$ than the non-competitive DAKO kit. This differ- ence in the CgA assay measurements must be taken into consideration when comparing results from clinical studies. In one study, including 45 patients with pheochromocytoma, where $\mathrm{CgA}$ was measured with the DAKO assay, the diagnostic sensitivity was only $71 \%$ (Boomsma et al. 1995). In a similar study of 50 patients with pheochromocytoma, where CgA was measured with a competitive assay, the sensitivity was $86 \%$ (Canale \& Bravo 1994). This is in line with another study, comprising 21 patients with pheochromocytoma, where CgA was measured with a competitive assay, giving a diagnostic sensitivity of 90\% (Stridsberg \& Husebye 1997).

There are three major causes for non-tumour-associated increases of plasma CgA, namely decreased renal function (Hsiao et al. 1990, Stridsberg \& Husebye 1997), type A gastritis (Borch et al. 1997) and treatment with PPIs (Sanduleanu et al. 2001). It is known that all these causes can give rise to substantial increases in CgA. Other causes mentioned are deteriorated liver function, increased activity in the sympathetic nervous system and inflammatory bowel disease. These, however, usually give only minor elevations of plasma CgA. Since all these causes are known factors that give increased levels of $\mathrm{CgA}$, we excluded the patients that were on PPI treatments before calculating the specificity. One could argue that we should have included the patients on PPI treatments, but excluding them more closely resembles the everyday clinical situation. Furthermore, these patients had increased levels of $\mathrm{CgA}$ in all three assays, so our interpretation of the results was not influenced.

Adrenocortical cancer is not considered to be a neuroendocrine disease, but in this study one of three patients had increased levels of CgA with the CIS and DAKO kits, while all three had elevated levels in the ED kit. It has previously been shown that corticosteroids can up-regulate the expression of CgA mRNA, which gives an increased production of CgA (Rozansky et al. 1994). The anatomical distribution of blood vessels in the adrenals makes the venous outflow from the cortical region run through the adrenal medulla. One can thus expect increased production of corticosteroids from an adrenocortical cancer to give rise to extremely high levels of corticosteroids locally in the adrenal medulla which, in turn, could stimulate increased production of $\mathrm{CgA}$. Based on this assumption, we also excluded the patients with adrenocortical cancers before calculating the specificity for the $\mathrm{CgA}$ kits. Instead, perhaps plasma CgA could also be a useful marker for adrenocortical cancers, albeit these tumours do not themselves express CgA.

We have concluded that $\mathrm{CgA}$ is an important tumour marker for all neuroendocrine tumours. However, different analytical methods give different results, which must be taken into consideration when comparing results from different clinical studies. A recognised international standard for CgA would be a step towards harmonisation, but antibody selection and construction of the assays will probably still influence the results. 


\section{Acknowledgements}

This work was accorded grants from the Swedish Cancer Foundation. We are grateful for the excellent technical assistance from Ulla-Britta Jansson and Inger Olsson.

\section{References}

Bajetta E, Ferrari L, Martinetti A, Celio L, Procopio G, Artale S, Zilembo N, Di Bartolomeo M, Seregni E \& Bombardieri E 1999 Chromogranin A, neuron specific enolase, carcinoembryonic antigen, and hydroxyindole acetic acid evaluation in patients with neuroendocrine tumors. Cancer 86 858-865.

Boomsma F, Bhaggoe UM, 't Veld AJMI \& Schalekamp MADH 1995 Sensitivity and specificity of a new ELISA method for determination of chromogranin $\mathrm{A}$ in the diagnosis of pheochromocytoma and neuroblastoma. Clinica Chimica Acta 239 $57-63$.

Borch K, Stridsberg M, Burman P \& Rehfeld JF 1997 Basal chromogranin A and gastrin concentrations in circulation correlate to endocrine cell proliferation in type-A gastritis. Scandinavian Journal of Gastroenterology 32 198-202.

Borglum Jensen TD, Holst JJ \& Fahrenkrug J 1991 Characterization of immunoreactive pancreastatin in porcine tissues. Scandinavian Journal of Clinical Laboratory Investigation 51 681-691.

Canale MP \& Bravo EL 1994 Diagnostic specificity of serum chromogranin-A for pheochromocytoma in patients with renal dysfunction. Journal of Clinical Endocrinology and Metabolism $\mathbf{7 8}$ 1139-1144.

Curry WJ, Johnston CF, Shaw C \& Buchanan KD 1990 Distribution and partial characterisation of immunoreactivity to the putative C-terminus of rat pancreastatin. Regulatory Peptides 30 207-219.

Franke WG, Pinkert J, Runge R, Bredow J, Wunderlich G \& Koch R 2000 Chromogranin A: an additional tumor marker for postoperative recurrence and metastases of medullary thyroid carcinomas? Anticancer Research 20 5257-5260.

Giovanella L, La Rosa S, Ceriani L, Uccella S, Erba P \& Garancini S 1999 Chromogranin-A as a serum marker for neuroendocrine tumors: comparison with neuron-specific enolase and correlation with immunohistochemical findings. International Journal of Biological Markers 14 160-166.

Hsiao RJ, Mezger MS \& O’Connor DT 1990 Chromogranin A in uremia: progressive retention of immunoreactive fragments. Kidney International 37 955-964.

Iacangelo AL \& Eiden LE 1995 Chromogranin A: current status as a precursor for bioactive peptides and a granulogenic/sorting factor in the regulated secretory pathway. Regulatory Peptides 58 65-88.
Laslop A, Doblinger A \& Weiss U 2000 Proteolytic processing of chromogranins. Advances in Experimental Medicine and Biology 482 155-166.

Nobels FR, Kwekkeboom DJ, Coopmans W, Schoenmakers CH, Lindemans J, De Herder WW, Krenning EP, Bouillon R \& Lamberts SW 1997 Chromogranin A as serum marker for neuroendocrine neoplasia: comparison with neuron-specific enolase and the alpha-subunit of glycoprotein hormones. Journal of Clinical Endocrinology and Metabolism 82 2622-2628.

Nobels FR, Kwekkeboom DJ, Bouillon R \& Lamberts SW 1998 Chromogranin A: its clinical value as marker of neuroendocrine tumours. European Journal of Clinical Investigation 28 431-440.

Portela-Gomes GM \& Stridsberg M 2001 Selective processing of chromogranin A in the different islet cells in human pancreas. Journal of Histochemistry and Cytochemistry 49 483-490.

Portela-Gomes GM, Grimelius L, Johansson H, Wilander E \& Stridsberg M 2001 Chromogranin A in human neuroendocrine tumors: an immunohistochemical study with region-specific antibodies. American Journal of Surgical Pathology 25 1261-1267.

Rozansky DJ, Wu H, Tang K, Parmer RJ \& O'Connor DT 1994 Glucocorticoid activation of chromogranin A gene expression. Identification and characterization of a novel glucocorticoid response element. Journal of Clinical Investigation 94 2357-2368.

Sanduleanu S, De Bruine A, Stridsberg M, Jonkers D, Biemond I, Hameeteman W, Lundqvist G \& Stockbrugger RW 2001 Serum chromogranin A as a screening test for gastric enterochromaffin-like cell hyperplasia during acid-suppressive therapy. European Journal of Clinical Investigation 31 802-811.

Stridsberg M 2000 Measurements of chromogranins and chromograninrelated peptides by immunological methods. Advances in Experimental Medicine and Biology 482 319-327.

Stridsberg M \& Husebye ES 1997 Chromogranin A and chromogranin $\mathrm{B}$ are sensitive circulating markers for phaeochromocytoma. European Journal of Endocrinology 136 67-73.

Stridsberg M, Hellman U, Wilander E, Lundqvist G, Hellsing K \& Öberg K 1993 Fragments of chromogranin A are present in the urine of patients with carcinoid tumours: development of a specific radioimmunoassay for chromogranin A and its fragments. Journal of Endocrinology 139 329-337.

Taupenot L, Remacle JE, Helle KB, Aunis D \& Bader M-F 1995 Recombinant human chromogranin A: expression, purification and characterization of the N-terminal derived peptides. Regulatory Peptides 56 71-88.

Received 2 January 2003

Accepted 29 January 2003 